\title{
Depressão na Atenção Primária à Saúde
}

\section{Depression in Primary Care}

Daniel Victor Arantes*

\section{Resumo}

Dos pacientes assistidos em ambulatórios gerais, 10\% a 25\% são portadores depressão ${ }^{1}$, representando a segunda causa de consulta, ainda que freqüentemente ignorada ou subdiagnosticada. Médicos generalistas, e não psiquiatras, tratam a maioria dos pacientes com sintomas depressivos ${ }^{2}$. A depressão ocupa o segundo lugar entre as doenças mais incapacitantes nos países ocidentais ${ }^{3}$. Na maioria dos países em desenvolvimento, a deficiência na saúde mental é ainda inaceitável, sendo meta da OMS aprimorar o diagnóstico e o tratamento da depressão em ambulatórios gerais em todo o mundo. Várias estratégias comprovam ser custo-efetivas, sendo as principais: educação médica continuada, maior participação da enfermagem e melhor integração entre atenção primária e secundária (especialista psiquiatra) ${ }^{4}$. Para depressão leve a moderada, estudos de meta-análise mostraram que há pouca diferença de efetividade entre as modalidades terapêuticas e entre antidepressivos, sendo a continuação da terapia o mais importante fator de escolha. Os melhores resultados são obtidos quando há aliança terapêutica entre o profissional de saúde e o paciente e um tratamento adequado é mantido por período suficiente ${ }^{5}$. Este estudo vem discorrer sobre a importância, diagnóstico e tratamento da depressão na atenção primária e propor estratégias para implementar uma rede efetiva de atendimento, articulada com a atenção secundária.

\section{Abstract}

Ten to 25\% of patients assisted in general outpatient care centers sufferfrom depression ${ }^{1}$. Although frequently ignored or underdiagnosed, depression is the second most frequent cause of consultations. In most cases, patients with symptoms of depression are assisted by general practitioners instead of psychiatrists ${ }^{2}$. Depression occupies the second place among the most disabling diseases in the western countries ${ }^{3}$. In most developing countries, mental healthcare is still unacceptably deficient, and one of the goals of the WHO is improving diagnosis and management of depression in general outpatient care centers all over the world. Several strategies prove cost-effective, mainly: continued medical education, more participation of the nursing personnel and better integration between primary and secondary care (psychiatrist) ${ }^{4}$. As demonstrated by metaanalytical studies, in the management of light or moderate depression there is little difference in efficiency between different treatment modalities and antidepressants. The most important factor is continuity of the treatment. The best results are achieved when there is therapeutic alliance

Palavras-chave: Depressão; Saúde Mental; Atenção Primária à Saúde.
Key Words: Depression; Mental Health; Primary Health Care.

\footnotetext{
* Médico de Família e Comunidade, Secretaria Municipal de Saúde, Prefeitura Municipal de Anápolis, GO, Brasil.
} 
between doctor and patient and when an adequate treatment is kept on for sufficient time $e^{5}$. This study discusses the importance, diagnosis and treatment of depression in primary care and proposes strategies for implementing a care network articulated with secondary care.

\section{Introdução}

O termo depressão, na linguagem corrente, tem sido empregado para designar tanto um estado afetivo normal (a tristeza), quanto um sintoma, uma síndrome e uma (ou várias) doença(s). Os sentimentos de tristeza e alegria "colorem" o fundo afetivo da vida psíquica normal. A tristeza constitui-se na resposta humana universal às situações de perda, derrota, desapontamento e outras adversidades. Cumpre lembrar que essa resposta tem valor adaptativo, do ponto de vista evolucionário, uma vez que, por meio do retraimento, poupa energia e recursos para o futuro. Por outro lado, constitui-se em sinal de alerta, para os demais, de que a pessoa está precisando de companhia e ajuda. As reações de luto, que se estabelecem em resposta à perda de pessoas queridas, caracterizam-se pelo sentimento de profunda tristeza, exacerbação da atividade simpática e inquietude. As reações de luto normal podem estender-se até por um ou dois anos, devendo ser diferenciadas dos quadros depressivos propriamente ditos. No luto normal, a pessoa usualmente preserva certos interesses e reage positivamente ao ambiente, quando devidamente estimulada. No luto, não se observa a inibição psicomotora característica dos estados melancólicos e os sentimentos de culpa limitam-se ao ato de não se ter feito todo o possível para auxiliar a pessoa que morreu - outras idéias de culpa estão geralmente ausentes. A depressão é considerada quando a tristeza perde sua função adaptativa e o humor segue autônomo persistentemente deprimido. Quando a tristeza ultrapassa o limiar da normalidade, perdendo seu papel adaptativo, e o humor persistentemente e autonomamente se torna deprimido, a depressão é estabelecida ${ }^{6}$.

Enquanto sintoma, a depressão pode surgir nos mais variados quadros clínicos, dentre os quais: transtorno de estresse pós-traumático, demência, esquizofrenia, alcoolismo e doenças clínicas. Ela pode ocorrer, ainda, como resposta a situações estressantes ou a circunstâncias sociais e econômicas adversas ${ }^{6}$.

Enquanto síndrome, a depressão inclui não apenas alterações do humor (tristeza, irritabilidade, falta da capacidade de sentir prazer, apatia), mas também uma gama de outros aspectos, incluindo alterações cognitivas, psicomotoras e vegetativas (sono, apetite) ${ }^{6}$.

Finalmente, enquanto doença, a depressão tem sido classificada de várias formas, na dependência do período histórico, da preferência dos autores e do ponto de vista adotado. Entre os quadros mencionados na literatura atual encontram-se: transtorno depressivo maior, melancolia, distimia, depressão integrante do transtorno bipolar tipos I e II, depressão como parte da ciclotimia, etc ${ }^{6}$.

\section{Epidemiologia}

Transtornos do humor estão entre as desordens que mais freqüentemente levam o paciente ao médico. Estima-se que de 15\% a 30\% dos adultos apresentem depressão clinicamente significativa em algum momento de sua vida, sendo cerca de $8 \%$ transtorno depressivo maior. Esses dados suportam os achados em estudos de Medicina de Família e Comunidade, em que depressão está no ranking das doenças mais prevalentes ${ }^{7}$. A prevalência em homens é estimada em 7\% a 12\% e, em mulheres, $20 \%$ a 25\%. Em ambulatórios de Atenção Primária, sua prevalência é estimada em $5 \%$ a $10 \%$ de todos os pacientes. Um recente estudo canadense evidenciou que os maiores índices de incidência do transtorno depressivo $(1,4 \%$ a $9,1 \%$ da população) ocorrem entre adultos jovens (idade entre 12 e 24 anos), e os mais baixos índices (1,3\% a 1,8\%) ocorrem entre pessoas acima de 65 anos. Os índices são significativamente mais altos em pessoas que apresentam determinadas condições médicas como obesidade, diabetes mellitus, câncer e história de infarto do miocárdio ${ }^{2,8}$.

Sabe-se que muitas pessoas que preenchem os critérios diagnósticos para depressão não seguem tratamento, o que acarreta graves conseqüências pessoais, sociais e econômicas. O transtorno depressivo é responsável por riscos 
significativos de morte e incapacidade. Cerca de 15\% dos pacientes com transtornos do humor morrem "por suas próprias mãos" e pelo menos $66 \%$ de todos os suicídios são precedidos por depressão. A depressão está associada à pobre produtividade no trabalho e afeta membros da família e cuidadores e; além disso, há crescente evidência de que crianças de mulheres deprimidas apresentam maiores índices de problemas comportamentais na escola e pior competência social. Ela é ainda causa líder de incapacidade e morte prematura de jovens entre 18 e 44 anos - e estimase que será a segunda causa de incapacidade entre todas as idades até $2020^{8}$.

A maioria dos pacientes deprimidos (60\%) recebe tratamento na Atenção Primária. Estima-se que 50\% das prescrições de antidepressivos sejam de clínicos, 30\% de psiquiatras e $20 \%$ de outras especialidades. Apesar da alta prevalência, há uma grande deficiência no diagnóstico e tratamento na prática geral ${ }^{8,9}$. Há recomendação formal para pesquisa sistemática de depressão na Atenção Primária, bem como tratamento e seguimento. Muitos instrumentos podem ser facilmente utilizados em ambulatórios gerais, tanto para screening quanto diagnóstico e tratamento ${ }^{1}$. Uma das escalas mais utilizadas para screening na Atenção Primária é o Beck Depression Inventory Scales - necessita de cerca de dez minutos para ser completada pelos pacientes e já foi validada em Português. Escore acima do ponto de corte indica necessidade de avaliação diagnóstica criteriosa quanto à depressão $\mathrm{O}^{2,8}$.

\section{Etiologia}

Depressão é compreendida como resultado da ruptura da neuroquímica normal do cérebro. Estudos nesta área têm levado ao desenvolvimento de medicamentos novos para o tratamento desse transtorno. Essas drogas parecem interferir nos neurotransmissores das monoaminas centrais. Vias neuronais norepinefrínicas centrais estão envolvidas em atividades de vigilância, motivação e geram níveis de energia. Essas vias neuronais compartilham um papel com vias serotoninérgicas, interferindo na ansiedade e irritabilidade. Vias serotoninérgicas parecem controlar a impulsividade ou ter um papel no controle dela e compartilham um papel com vias dopaminérgicas no apetite, no sexo e na agressividade. Vias dopaminérgicas também estão envolvidas na motricidade, cognição e memória, recompensa e pragmatismo. Nosso crescente conhecimento nesta área tem ajudado na compreensão da etiologia e farmacoterapia dos transtornos do humor. Sabe-se que o tratamento antidepressivo de "longa duração" (em especial além de 30 dias) resulta em ativação sustentada do 3-5-monofosfato de adenosina cíclico (ou AMPc) em regiões específicas do cérebro, que desencadeia reações que regulam e ativam genes específicos, favorecendo restabelecimento da via hipocampal pelo aumento do fator neurotrófico cerebral, uma substancia endógena neuroprotetiva. Estresse psicológico, importante na patogênese da depressão, pode reduzir a produção de o fator neurotrófico cerebral e levar à hipotrofia neuronal hipocampal. Adicionalmente, uma série de estudos de imagem cerebral evidenciam consistentemente redução da atividade neuronal no córtex prefrontal dorsolateral, diretamente relacionado à severidade da depressão. Conclui-se então que uma hipótese atual sobre o desenvolvimento do transtorno depressivo leva em conta a indução de vulnerabilidade por estresse ambiental, que, aliado a suscetibilidade genética própria de cada um, desencadeia uma cascata de mecanismos intracelulares que interferem em fatores neurotróficos específicos necessários para a sobrevivência e funcionamento de determinados neurônios cerebrais. Também há evidencia de que não apenas antidepressivos, mas também eletroconvulsoterapia e psicoterapia podem interferir beneficamente no crescimento neuronal e metabolismo cerebral regional ${ }^{8}$.

\section{Sinais e Sintomas}

\subsection{Sintomas psíquicos}

- Humor depressivo: sensação de tristeza, autodesvalorização e sentimentos de culpa.

- Redução da capacidade de experimentar prazer na maior parte das atividades, antes consideradas como agradáveis.

- Fadiga ou sensação de perda de energia. A pessoa pode relatar fadiga persistente, mesmo sem esforço físico, e as tarefas mais leves parecem exigir esforço substancial. O 
tempo para a execução das tarefas torna-se mais lento.

- Diminuição da capacidade de pensar, de se concentrar ou de tomar decisões, as quais, antes, eram quase automáticas.

\subsection{Sintomas fisiológicos}

- Alterações do sono (mais freqüentemente insônia, podendo ocorrer também hipersonolência). A insônia é, mais tipicamente, intermediária (acordar no meio da noite, com dificuldades para voltar a conciliar o sono) ou terminal (acordar mais cedo pela manhã).

- Alterações do apetite (mais comumente perda do apetite, podendo ocorrer também aumento dele).

- Redução do interesse sexual

\subsection{Evidências comportamentais}

- Retraimento social.

- Crises de choro.

- Comportamentos suicidas.

- Retardo psicomotor e lentificação generalizada, ou agitação psicomotora.

Deve-se ainda lembrar, no diagnóstico das depressões, que algumas vezes o quadro mais típico pode ser mascarado por queixas proeminentes de dor crônica (cefaléia, dores vagas no tórax, abdômen, ombros, região lombar, etc.). A ansiedade está freqüentemente associada. Em idosos, principalmente, as queixas de caráter hipocondríaco costumam ser muito comuns ${ }^{6}$.

\section{Diagnóstico}

O conceito de depressão maior, como aparece no DSM-IV, é excessivamente abrangente e, por isso mesmo, pouco preciso. Ela abarca provavelmente uma gama muito heterogênea de condições, que vão desde as fronteiras da normalidade (reações de luto ou tristeza normal) até aquelas formas mais graves de depressão, para as quais provavelmente concorrem fatores mais biológicos (adquiridos e/ou geneticamente determinados). Para o diagnóstico de Transtorno Depressivo Maior, de acordo com o DSM-IV, basta que a pessoa apresente "humor deprimido ou perda de interesse ou prazer, durante um período de duas semanas", mais quatro sintomas de uma lista de nove (ou mais três sintomas, se os dois primeiros estiverem presentes). Assim, por exemplo, se uma moça que brigou com o namorado apresentar tristeza e perda de energia por 15 dias, além de mais três sintomas, como insônia, perda de energia e capacidade diminuída de se concentrar, terá preenchido critérios para Transtorno Depressivo Maior. Um conceito tão amplo certamente não contribui para que se testem hipóteses sobre a etiologia das depressões, resposta a tratamentos biológicos etc. Não serve tampouco para auxiliar na decisão de se medicar, ou não, a pessoa que preencha tais critérios. Já o conceito de melancolia é muito mais preciso e, por essa razão, tem maior valor preditivo quanto, por exemplo, à resposta terapêutica a antidepressivos e ao eletroconvulsoterapia ${ }^{6}$.

O transtorno depressivo é diagnosticado utilizando métodos similares para condições clínicas. Há necessidade de coleta detalhada e cuidadosa da história familiar do paciente e de seus familiares, exame clínico e investigações especiais, se necessário ${ }^{6}$.

O conhecimento dos fatores de risco para depressão possibilita alto índice de suspeição. Homens apresentando sintomas físicos, fadiga ou redução de energia, insatisfação com o trabalho ou história de episódio depressivo prévio são os mais prováveis candidatos a um episódio atual. Mulheres com distimia, ataques de pânico, consultas freqüentes (mais de sete) também são ${ }^{6}$.

Algumas técnicas podem ser usadas para melhor detecção:

Técnica de entrevista - Por poucos minutos é necessário escutar ativamente as queixas e explorar a natureza destas, facilitando ao paciente descrever detalhes.

Pesquisa de depressão - Um teste de duas questões tem sido considerado útil no reconhecimento do transtorno na Atenção Primária: (1) Deste o último mês, você freqüentemente tem se sentido triste, deprimido ou sem esperança? (2) Desde o último mês, você tem freqüentemente sentido pouco interesse ou prazer pelas coisas em geral? No caso de respostas afirmativas, o transtorno depressivo deve ser sistematicamente pesquisado.

Questões para esclarecer a natureza e extensão dos 
sintomas depressivos e disfunções associadas:

Perda de energia - Você sente perda de energia ou vigor? Redução de concentração - Você acha que pode ler um artigo no jornal ou assistir televisão da mesma forma que sempre?

Auto-confidência - O quanto tem sido responsável com os compromissos com outros ou administrando atividades do dia-a-dia?

Redução da auto-estima - Qual sua opinião a respeito de si mesmo comparado com outros? Você se acha menos competente? Você se sente inferior ou pior que outros?

Desesperança - Como você vê o futuro? Tudo parece muito difícil?

Idéias de morte - Você, muitas vezes, sente que a vida não vale a pena? Ou você não se importaria se não acordasse pela manhã? Você tem pensado em se ferir ou até em suicídio?

Provável causa da depressão - A que você atribui a causa de sua aflição? O que o fez tão infeliz nesses dias? Ocorreu algo de ruim com você ou sua família nos dias que precederam seus sintomas?

Todas as respostas positivas devem ser seguidas por questões a respeito, como: Qual a intensidade e duração dos sintomas? Com que freqüência eles tem ocorrido? Quanto tem interferido os sintomas nas atividades diárias? Qual o tipo de problema? Quais esferas da vida têm sido afetadas - social, pessoal, familiar, ocupacional, etc.?

Entrevistando um paciente clinicamente julgado como deprimido que nega ter o transtorno:

- Como você descreve seu humor nas últimas semanas?

- Descreva-me um pouco como você tem se sentido ultimamente?

- Que tipo de coisas você gosta de fazer quando esta fora do trabalho? Você tem feito essas coisas? Você tem sido capaz de aproveitá-las como antes?

- Quais eram seus interesses habituais? Você tem perdido interesse nas coisas ultimamente?

\section{Diagnósticos diferenciais}

É importante lembrar que outros transtornos do humor podem apresentar-se com síndrome depressiva, sendo importante pesquisa sistemática de diagnósticos diferenciais por necessidade de tratamento distinto.

Depressão menor - Mais prevalente na prática clínica que a depressão maior, o paciente apresenta por mais de duas semanas de dois a cinco sintomas descritos acima. Os pacientes nesta condição podem ter menos sintomas vegetativos (apetite, variação de humor diurna) e mais sintomas subjetivos ("autoculpa", preocupação, irritabilidade, letargia). O fracasso em tratar apropriadamente esta condição pode ter ao longo do tempo grande impacto na saúde, posição funcional e qualidade de vida, além de custos para os pacientes. A noção que a depressão menor não necessita de tratamento é errônea, sendo as melhores opções para tratá-la: antidepressivos e psicoterapia ${ }^{10}$.

Distimia - Ocorrência de $8 \%$ ao longo da vida na população geral ${ }^{8}$ caracterizada por humor cronicamente deprimido (dois ou mais anos) em um nível mais leve sem comprometimento significativo das atividades diárias ${ }^{11}$.

Transtorno Bipolar do Humor - Ocorrência em cerca de $2 \%$ da população geral ao longo da vida ${ }^{8}$, sendo que de $12,5 \%$ a $30 \%$ dos pacientes diagnosticados como deprimidos apresentam na verdade transtorno bipolar do humor. Clínicos inevitavelmente encontraram pacientes bipolares, geralmente durante o episódio depressivo. $\mathrm{Na}$ maioria desses pacientes, a elevação do humor é representada pela hipomania (Bipolar tipo II), havendo necessidade de questionamento específico a respeito da história do paciente ou se estes permaneceram subdiagnosticados. Os episódios depressivos podem ser mais refratários, com mais sintomas físicos e possibilidade de virada maníaca, havendo necessidade de estabilizador do humor (carbonato de lítio, ácido valpróico e carbamazepina) antes da terapia antidepressiva ${ }^{12}$. Considerar referir para o psiquiatra.

\section{Tratamento}

Assim que a depressão é diagnosticada, o tratamento deve ser instituído de forma adequada e os antidepressivos são a principal forma de tratamento, embora outras opções estejam disponíveis. 


\section{Tratamento medicamentoso}

Escolha da medicação - Não há diferença de eficácia entre antidepressivos, mas, sim, de modo de ação e efeitos colaterais. A escolha dever ser feita com base em diversos dados referentes ao paciente (idade, comorbidades, resposta anterior) e ao fármaco (custo, efeitos colaterais e disponibilidade). Antidepressivos tricíclicos são bem conhecidos por sua eficácia, no entanto os efeitos anticolinérgicos tendem a limitar seu uso em idoso e pacientes com comorbidades. Os inibidores seletivos da recaptação da serotonina (ISRS), por outro lado, mostraram-se seguros nesses pacientes. Antidepressivos com perfil sedativo, como doxepina, trazodona e mirtazapina, devem ser de escolha em pacientes agitados ou com insônia ${ }^{11}$. Na Atenção Primária, baixas doses se mostraram efetivas no acompanhamento da maioria dos pacientes $^{13}$.

Início do tratamento - Informar o paciente a respeito do tratamento medicamentoso e iniciar a mínima dose efetiva do medicamento escolhido. Não é recomendado iniciar múltiplos fármacos ou em subdose. No entanto, pode ser prudente iniciar tratamento com metade da dose habitual em pacientes debilitados, crianças e adolescentes (risco de suicídio) e idosos ${ }^{11}$.

Monitoramento - O efeito antidepressivo se inicia entre duas a quatro semanas após o início do tratamento. O paciente deve ser monitorado neste período quanto à resposta e aos eventos adversos. A decisão de aumentar a dose somente deve ser considerada após duas a quatro semanas e o medicamento não deve ser descontinuado (se tolerado) até que se complete seis a oito semanas. Após estabilização clínica, o paciente pode ser acompanhado por um período mensal. É essencial questionar a família a respeito do uso adequado do medicamento pelo paciente, visto que a não-aderência é comumente uma das razões de não resposta ${ }^{11}$.

Duração do tratamento - Após melhora do paciente, é necessário manter o tratamento por um período de pelo menos um ano para prevenir recaídas. Antidepressivos são freqüentemente prescritos na Atenção Primária, no entanto por períodos curtos de tempo. Este tipo de prescrição freqüentemente leva a cronicidade do quadro ${ }^{11}$.

Educação do paciente e da família - Os pacientes e os membros da família freqüentemente carregam estigmas acerca da depressão e seu tratamento. Durante este cuidado algumas informações mínimas são necessárias, como por exemplo: (1) depressão é doença comum e há tratamento efetivo disponível; (2) depressão não é fraqueza ou preguiça; (3) depressão pode afetar a capacidade de decisão; (4) suporte emocional e prático de familiares e amigos é recurso muito válido; (5) antidepressivos não provocam dependência ${ }^{11}$.

O que referir ao psiquiatra - As principais indicações são: (1) paciente expressa ativa intenção suicida ("Eu quero me matar!'); (2) falha de resposta aos medicamentos em doses e período adequados; (3) comorbidades como transtorno obsessivo compulsivo ou abuso de substancias; (4) pacientes com doença grave, com sintomas psicóticos ou severa agitação; (5) pacientes preferindo psicoterapia, como terapia cognitiva comportamental. É necessário explicar ao paciente e à família a razão pela qual se decidiu pela referência ${ }^{11}$.

\section{Considerações Especiais Crianças e Adolescentes}

Há evidência insuficiente para recomendar a pesquisa rotineira de depressão em crianças e adolescentes, embora clínicos devam estar alerta sobre sinais e sintomas em indivíduos jovens ${ }^{2}$.

A incidência de adolescentes com depressão aumenta de $1,5 \%$ para $8 \%$ de amostras de comunidade para ambulatoriais, respectivamente. Apesar do risco de depressão ser equivalente em garotos e garotas pré-púberes, o risco de garotas aumenta após puberdade. Os índices mais alarmantes são de grávidas e pós-parturientes, provavelmente devido ao impacto do recém-nascido. Sintomas de depressão têm sido identificados em mais de 68\% de adolescentes grávidas e $47 \%$ de pós-parturientes ${ }^{14}$. Crianças abaixo de seis anos raramente apresentam o transtorno, sendo a incidência em torno de $2 \%$ em pré-adolescentes ${ }^{2}$. Há crescente evidência de que crianças de mulheres deprimidas apresentam maior risco de problemas escolares e comportamentais, além de 
baixos níveis de competência social em comparação com as que não tem ${ }^{8}$. Indivíduos com inicio precoce de depressão têm alta carga genética ou familiar predisponente. Independente do fator envolvido, é importante o trabalho familiar. Uma das formas de avaliar depressão em crianças e adolescentes é avaliar familiares para depressão, sendo particularmente importante avaliar adolescentes ${ }^{1}$.

Depressão interfere no desenvolvimento dos adolescentes - que não vão bem na escola, têm dificuldades nos relacionamentos, mais freqüentemente cometem suicídio, abusam de álcool e drogas e estão sob maior risco de desenvolver depressão na idade adulta ${ }^{14}$. Dificuldades podem persistir por longos períodos após o episódio terse resolvido. $\mathrm{O}$ diagnóstico pode ser difícil por problemas de comunicação - parece ser raro em crianças abaixo de seis anos. Aproximadamente $2 \%$ de crianças em idade escolar fundamental apresentam depressão, e este índice cresce para $8 \%$ em adolescentes ${ }^{2}$.

O uso de serviços de saúde mental por adolescente é baixo. Crianças e adolescentes com transtornos mentais freqüentemente permanecem subdiagnosticados e não recebem tratamento de fonte alguma. Profissionais de saúde de ambulatórios gerais são, geralmente, a porta de entrada para os serviços especializados, mas estudos têm demonstrado que não são capazes de reconhecer depressão em metade dos pacientes. Estes profissionais devem rotineiramente pesquisar depressão em garotas adolescentes como diagnóstico diferencial de desordens somáticas ${ }^{14}$.

Feito o diagnóstico, é de suma importância determinar o risco de suicídio e solicitar auxílio imediatamente para adolescentes de alto risco. Tanto medicações quanto terapia são indicados. Inibidores seletivos da receptação da serotonina (ISRS) são os medicamentos de escolha por serem associados a menores riscos e toxicidade, menos efeitos colaterais, além de serem mais fáceis de se usar. Devem ser iniciados em dose baixa (tipicamente metade da dose do adulto) e aumentados lentamente (incremento equivalente à metade da dose inicial), e mantido por um período livre de sintomas de no mínimo seis meses antes que seja descontinuado. Se houver episódio prévio de depressão, terapia de longa duração é preferida. Importante frisar que algumas indústrias farmacêuticas fazem alerta para o risco de uso de alguns medicamentos em crianças e adolescentes Wyeth Pharmaceuticals para Effexor e GlaxoSmithKline para Paxil) ${ }^{14}$.

Psicoterapia interpessoal tem demonstrado ser eficaz no tratamento de depressão em garotas adolescentes, provavelmente grande papel de desajustes em relacionamentos interpessoais no desenvolvimento da depressão, devendo ser então, se possível, encaminhados para este tipo de tratamento. Terapia cognitiva também se mostrou efetiva e terapia familiar freqüentemente está indicada ${ }^{14}$.

Quanto a gestantes, profissionais de saúde da Atenção Primária devem escolher entre tratar, co-tratar ou referir estas adolescentes para serviços de saúde mental. Alguns antidepressivos, em especial ISRS, são considerados de baixo risco na gestação, no entanto dados precisos ainda não estão disponíveis ${ }^{14}$.

\section{Idosos}

Há pouca evidência sobre efetividade de tratamentos antidepressivos na população idosa acompanhada na Atenção Primária, particularmente aqueles com transtornos depressivos mais leves. Idosos em geral tomam mais medicações, sendo antidepressivos mais freqüentemente contra-indicados. Neste caso, intervenções não-farmacológicas podem ser bem indicadas, necessitando de mais estudos validar seu uso ${ }^{15}$

Antidepressivos são efetivos em idosos. A regra "comece baixo, vá devagar" é adequada e o uso de novos agentes, em particular aqueles com mínima inibição de CYP450, é apropriado e seguro, mais que antidepressivos tricíclicos ou $\mathrm{IMAO}^{8}$.

A prevalência de depressão em adultos acima de 65 anos de idade aumenta de 7\% para 36\% em pacientes ambulatoriais e 40\% em hospitalizados. Mais de 50\% de pacientes com doença de Alzheimer ou Parkinson desenvolvem transtornos depressivos e seus cuidadores também estão em maior risco. Sinais de depressão mais comuns nos idosos que em outras populações incluem diminuição 
de cuidados pessoais, irritabilidade e retardo psicomotor. Diagnóstico e tratamento precoces melhoram qualidade de vida e status funcional e podem impedir morte prematura. A aplicação de escalas em idosos pode estar dificultada por déficits visuais e cognitivos. Uma questão de um item para screening pode ser tão efetiva quanto outros testes e poupar tempo: "Você se sente triste ou deprimido?" 2 .

Os resultados do presente estudo evidenciaram que a EDG-15 é um instrumento válido para o rastreamento dos transtornos do humor, sugerindo que a escala pode ser utilizada na prática clínica para a identificação destes transtornos na população geriátrica ambulatorial brasileira. O ponto de corte $5 / 6$, sugerido inicialmente pelos autores que traduziram a escala para o português, e corroborado por outros autores, mostrou-se adequado também na atual amostra. Dada a alta prevalência, suas dificuldades diagnósticas e o baixo nível de reconhecimento clínico dos transtornos do humor, a EDG-15, se adequadamente utilizada, pode se tornar um instrumento valioso de saúde pública na identificação e no controle destas condições em ambientes não-especializados ${ }^{16}$.

\section{Recomendações para Tratamento da Depressão na Atenção Primária}

A maioria dos pacientes deprimidos deve ser tratada em ambulatórios gerais, pelo clínico somente, ou (para a depressão mais grave) em conjunto com psiquiatra.

- Planos de tratamento sempre devem ser fundamentados na avaliação completa do quadro, incluindo: tipo; gravidade e duração do episódio depressivo; presença de qualquer estressor que contribua para o episódio.

- Para a depressão leve a moderada, estudos de meta-análise mostraram que há pouca diferença na eficácia relativa entre os diversos tratamentos, e a continuação da terapia é mais importante do que a escolha do tratamento inicial.

- Os melhores resultados são alcançados quando uma boa aliança terapêutica é formada entre um profissional e o paciente, e o tratamento adequado é fornecido por período de longo prazo. Para intervenções farmacológicas, o tratamento deve se estender para:

- pelo menos um ano no caso do primeiro episódio depressivo;

- pelo menos dois anos, no caso de episódios recorrentes ou onde há permanência de fatores de riscos $\operatorname{associados}^{5,17}$.

\section{Intervenções para Melhorar a Assistência à Depressão na Atenção Primária}

Gestores políticos e de saúde pública devem adotar estratégias para melhorar o tratamento de depressão na atenção, sendo esta estratégia considerada custo-efetiva por diversos estudos e demonstrou ter efeito positivo na economia de um modo geral, por aumento de produtividade e redução de absenteísmo ${ }^{18,19,20}$.

O tratamento da depressão na Atenção Primária pode ser comprometido por limitações da eficácia, aceitabilidade e pouca disponibilidade de tratamentos existentes. As melhoras no tratamento podem originar-se das ações individuais de clínicos e da disponibilização de novas formas do tratamento. Clínicos podem assegurar tratamento adequado mantendo-se atualizados a respeito do transtorno depressivo, sempre atentos para o diagnóstico e seguindo recomendações atualizadas de tratamento disponibilizadas por meio de treinamentos, discussões com colegas e leitura ${ }^{19}$.

Informações sobre o transtorno e estratégias de auto-ajuda, oferecidas de forma verbal ou escrita e dirigidas a pacientes e cuidadores, melhoram a aderência e os resultados do tratamento. Dentro da Atenção Primária, a provisão de tratamentos psicológicos simplificados por outros membros da equipe, como enfermeiras, deve ser instigada e avaliada ${ }^{19}$

Os coordenadores e gestores da Atenção Primária devem apoiar tais iniciativas, tanto oferecendo suporte aos profissionais de saúde quanto estabelecendo contato com agências governamentais e não-governamentais solicitando suporte estratégico e de pesquisa ${ }^{19}$.

Rede especializada deve oferecer suporte executivo e treinamento aos profissionais da Atenção Primária, além 
de tratamento de casos resistentes.

Em vista da alta prevalência e incidência de depressão na atenção e da escassez de recursos, supõe-se que modestos investimentos na assistência a pacientes deprimidos possam resultar em benefício consideráve ${ }^{19}$.

\section{Referências}

1. Bromberger JT. A psychosocial understanding of depression in women: for the primary care physician. J Am Med Womens Assoc. 2004 Summer; 59(3):198-206.

2. Sharp LK, Lipsky MS. Screening for depression across the lifespan: a review of measures for use in primary care settings. Am Fam Physician. 2002 Sep 15;66(6):1001-8.

3. Murray CJ, Lopez AD. Global mortality, disability, and the contribution of risk factors: Global Burden of Disease Study. Lancet. 1997 May 17;349(9063):1436-42.

4. Gilbody S, Whitty P, Grimshaw J, Thomas R. Educational and organizational interventions to improve the management of depression in primary care: a systematic review. JAMA. 2003 Jun 18;289(23):3145-51.

5. Ellis PM, Smith DA; beyond blue: the national depression initiative. Treating depression: the beyondblue guidelines for treating depression in primary care. "Not so much what you do but that you keep doing it". Med J Aust. 2002 May 20;176 Suppl:S77-83.

6. Del Porto JA. Conceito e diagnóstico. Rev Bras Psiquiatr. 1999. Maio; 21 (supl.1): 06-11.

7. Feightner JW, Worrall G. Early detection of depression by primary care physicians. CMAJ. 1990 Jun 1;142(11):121520.

8. Remick RA. Diagnosis and management of depression in primary care: a clinical update and review. CMAJ. 2002 Nov 26;167(11):1253-60.

9. Frank RG, Huskamp HA, Pincus HA. Aligning incentives in the treatment of depression in primary care with evidence-based practice. Psychiatr Serv. 2003 May; 54(5):682-7.

10. Banazak DA. Minor depression in primary care. J Am Osteopath Assoc. 2000 Dec; 100(12):783-7.

11. Sengupta S. Diagnosis of depression in general prac- tice. Indian J Med Sci. 2005 May; 59(5):217-25.

12. Manning JS, Connor PD, Sahai A. The bipolar spectrum: a review of current concepts and implications for the management of depression in primary care. Arch Fam Med. 1998 Jan-Feb;7(1):63-71.

13. Arroll B, Macgillivray S, Ogston S, Reid I, Sullivan F, Williams B, Crombie I. Efficacy and tolerability of tricyclic antidepressants and SSRIs compared with placebo for treatment of depression in primary care: a meta-analysis. Ann Fam Med. 2005 Sep-Oct;3(5):449-56.

14. Logsdon MC. Depression in adolescent girls: screening and treatment strategies for primary care providers. J Am Med Womens Assoc. 2004 Spring;59(2):101-6.

15. Freudenstein U, Jagger C, Arthur A, Donner-Banzhoff N. Treatments for late life depression in primary care-a systematic review. Fam Pract. 2001 Jun;18(3):321-7.

16. Paradela EMP, Lourenço, RA, Veras, RP. Validação da escala de depressão geriátrica em um ambulatório geral. Rev Saúde Pública. 2005 Dez; 39 (6): 918-923.

17. Strategies for effective management of depression in primary care. Based on a presentation by Michael S. Klinkman, MD. Am J Manag Care. 1999 Sep;5(13 Suppl):S783-8; discussion S788-93. [No authors listed]. 18. Rost K, Pyne JM, Dickinson LM, LoSasso AT. Costeffectiveness of enhancing primary care depression management on an ongoing basis. Ann Fam Med. 2005 JanFeb;3(1):7-14.

19. Moore RG. Improving the treatment of depression in primary care: problems and prospects. Br J Gen Pract. 1997 Sep;47(422):587-90.

20. Rost K, Smith JL, Dickinson M. The effect of improving primary care depression management on employee absenteeism and productivity. A randomized trial. Med Care. 2004 Dec;42(12):1202-10.

\section{Endereço para correspondência:}


Daniel Victor Arantes

Praça James Fanstone, 10 / $2^{\circ}$ andar

Anápolis, GO

CEP 75020-330

\section{Endereço eletrônico:}

d_arantes@msn.com 